\title{
Kinematic and Electromiographic Analysis of the Water Polo Crawl While Leading the Ball: A Pilot Study
}

\author{
F. Pereira ${ }^{1}$, P. Figueiredo ${ }^{1}$, P. Gonçalves ${ }^{1}$, S. Pereira ${ }^{1,2}$, J.P. Vilas-Boas ${ }^{1}$, R.J. Fernandes ${ }^{1, *}$ and \\ L. Machado ${ }^{1}$ \\ ${ }^{1}$ University of Porto, Faculty of Sport, Cifi2d, Porto, Portugal \\ ${ }^{2}$ State University of Santa Catarina, Florianópolis, Brasil
}

\begin{abstract}
Our aim was to conduct a kinematic and electromyographic characterization of the water polo front crawl while leading the ball technique. A high level male water polo player performed an experimental protocol of $3 \times 15 \mathrm{~m}$ at front crawl while leading the ball (with rest intervals of $2 \mathrm{~min}$ between repetitions). Each repetition was performed in acceleration. One complete stroke cycle was analyzed for each repetition of $15 \mathrm{~m}$ using surface electromyography to analyse the biceps femoris, gastrocnemius medialis, tricips brachii, biceps brachii, deltoideus posterior and rectus femoris muscles. The protocol was also recorded by 2 surface and 4 underwater cameras in order to assess centre of mass (CM) velocity during the cycle and its fluctuations. EMG signal was digital filtered $(35-500 \mathrm{~Hz})$, full-wave rectified, calculated the linear envelope, normalized to the maximal isometric voluntary contraction and integrated (iEMG). The fluctuations of $\mathrm{CM}$ velocity were assessed though the coefficient of variation of the instantaneous velocity. Results showed that tricips brachii and gastrocnemius medialis were the most active muscles in the beginning of the cycle and that gastrocnemius medialis, deltoideus posterior and tricips brachii displayed the highest values (and biceps brachii the lower values) of maximum electric activity and iEMG. It was observed an increase in the velocity of the CM at the beginning of the cycle, decreasing slightly afterwards, implying a reduced coefficient of variation (16.8). It should be evidenced the different involvement of the studied muscles in the cycle of front crawl while leading the ball and the consequent differences in the CM velocity.
\end{abstract}

Keywords: Biomechanics, centre of mass, crawl leading the ball, electromyography, kinematics, water polo.

\section{INTRODUCTION}

One of the most important skills in water polo is the front crawl technique, which, from all the swimming volume that is done during a match, occupies $89.5 \%$, being a distance of 280 to $430 \mathrm{~m}$ (about $27.9 \%$ ) performed at maximum or sub-maximum intensity [1]. During the match, the front crawl technique is conducted in different variants one of which is the front crawl technique while leading the ball, being used during $6.6 \%$ of the match duration. Complementarily, electromyography (EMG) applied to swimming could express the dynamic evolvement of the specific muscles responsible for the propulsion of the body through the water. Surface EMG assesses the electric potential of the working muscles and provides valuable information to better understand the swimming actions. However, the water polo player does not move at a constant velocity, existing accelerations and decelerations of the centre of mass $(\mathrm{CM})$, even in a single stroke cycle [2], which are the effect of non-constant resistive and propulsive forces acting upon the athlete's body. These velocity variations within a stroke cycle are known as intracyclic speed fluctuations (dv) [3], being considered as an indicator of the inverse of swimming efficiency [4].

*Address correspondence to this author at the Faculty of Sport, Porto University, Portugal; Tel: +351 225074763; E-mail: Ricfer@fade.up.pt

\section{METHODS}

A high level male water polo player (21 years old, $75 \mathrm{~kg}$, $178.5 \mathrm{~cm}$ and $8.7 \%$ of fat mass) performed an experimental protocol of $3 \times 15 \mathrm{~m}$ at front crawl while leading the ball (with rest intervals of 2 min between repetitions). Each repetition was performed in acceleration.

Surface EMG was used to analyse the muscles biceps femoris (BF), gastrocnemius medialis (GM), tricips brachii (TB), bíceps brachii (BB), deltoideus posterior (DP) and rectus femoris $(\mathrm{RF})$. The player's skin was prepared and the active bipolar electrodes were placed accordingly with the European Recommendations for Surface Electromyography [5]. The electrodes were water-proofed using adhesives (Tegaderm3M®) and silver tape. The player used a Fast Skin ${ }^{\circledR}$ swimsuit, with a cable entrance opened in the lateraldorsal position. Over the water, at a $2 \mathrm{~m}$ height, a steel cable was extended with a pulley solution to fix the EMG cables. The active electrodes used a pre-amplifier (AD621BN), with a 100 gain and a CMRR of $110 \mathrm{~dB}$ [6]. The pre-amplified signals were transmitted through $25 \mathrm{~m}$ cables to the main amplifier, where the signals were conditioned and further amplified $11 x$, in a total amplification of 1100 [7]. The signals were acquired by an A/D converter (BIOPAC Systems, Inc.) with a sampling frequency of $1000 \mathrm{~Hz}$. The numerical treatment of the signals was conducted using MATLAB software. The steps for the treatment of the EMG signal were: (i) digital filtering, pass-band of 35- 
$500 \mathrm{~Hz}$; (ii) removal of the common component (DC offset); (iii) full-wave rectification; (iv) linear envelope; (v) normalization of the signal for the maximum value of maximal isometric voluntary contraction and (vi) integral of treated signal (iEMG).

One complete stroke cycle was analyzed for each repetition of $15 \mathrm{~m}$, being monitored while the swimmer passed through a specific calibrated space: a cube of $27 \mathrm{~m}^{3}$, with 12 calibration points. The protocol was recorded by 2 surface and 4 underwater fixed cameras (Sony® DCR-HC42E), at $50 \mathrm{fps}$, and the video images were manually digitized with APASystem (Ariel Dynamics, USA) coupled with Zatsyiorsky anthropometric biomechanical model, adapted by de Leva [8]. Twenty reference anatomical points and a zero mass point for the ball were used. The synchronisation of the images was obtained using two lights fixed to the calibration volume. The 3D reconstruction used Direct Linear Transformation [9] procedure and a low-pass filter of $5 \mathrm{~Hz}$, as proposed by Winter [10].

\section{RESULTS}

Fig. (1) (A panel) shows an increase in the velocity of the $\mathrm{CM}$ at the beginning of the cycle, decreasing slightly afterwards, and keeping the values steadier later. These facts imply a reduced coefficient of variation (16.8\%), showing a low variability in the average curve of the CM speed. Additionally, regarding EMG assessment, it was verified that in the beginning of the cycle the more active muscles were the TB and the GM (B panel). The values of the variation's coefficient of the linear envelope of the EMG signals were $100.4 \%$ (BF), $84.9 \%$ (GM), $68.1 \%$ (TB), $107.3 \%$ (BB), 91.2\% (DP), $95.3 \%$ (RF).
Fig. (2) shows the mean values of maximum electric activity and iEMG for the studied muscles. It is visible that GM, DP and TB display the highest values of maximum electric activity (TB presents smaller variation's coefficient relatively to the DP evidencing lower variability in the analyzed cycles). The BB reveals the lower values of maximum electric activity and iEMG. Considering iEMG, TB and GM presented a higher muscular involvement (GM presented higher variation's coefficient).

\section{DISCUSSION}

To the best of our knowledge no biomechanical study was conducted aiming to study the water polo front crawl while leading the ball. The data obtained for the CM velocity in this specific technique displays one curve with a peak velocity, which shows that there is not a period of deceleration between the underwater actions of the arm. The TB muscle presents high levels of activity at initial stages of the stroke, which is coincident with the elbow's extension in the entry of the hand and beginning of the downsweep. The TB's activity is high during almost all the underwater stroke, decreasing its activity during the upsweep and recuperation of the arm, which is executed with elbow's flexion. In the final of the cycle, it is observed a new increase of EMG activity when the movement of elbow's extension to prepare new entry of the hand. The high levels of activity presented by GM, corresponding to EMG peaks, match with the upbeat (when the foot is on its plantar flexion - fin effect), being possible to observe a decrease of its activity in the downbeat. The BF also presents an increase of its EMG activity in the upbeat and a decrease in the beginning of the downbeat (in the final of the cycle, new moderate and constant increase of


Fig. (1). Mean velocity curve of the CM (A panel) and linear envelope of the EMG signals (B panel).



Fig. (2). Mean and SD values of the maximum normalized electric activity (left) and normalized iEMG (right) for the studied muscles during a stroke cycle of front crawl while leading the ball. 
EMG activity, resulting from the new upbeat can be observed). The higher activity values of the RF occur when the knee is in extension. The DP presents three activity peaks, corresponding to the downsweep of the arm, while the upsweep and the recovery phases occur following the action of this muscle. The BB presents higher values of EMG activity during the arm stroke when the elbow is in flexion, corresponding to the insweep, upsweep and recovery of the arms.

After observing the values of the variation's coefficient relatively to the EMG activation of the referred muscles, we can conclude that there exists an very large variability in the activation during the analysed cycle.

\section{REFERENCES}

Dopsaj M, Matković I. Motorička aktivnost vaterpolista u toku igre (Motorical activity of water polo players during the game). Fizička Kult 1994; 48 (4): 339-46.

[2] Barbosa T, Keskinen K, Fernandes R, Colaco P, Lima A, VilasBoas JP. Energy cost and intracyclic variation of the velocity of the centre of mass in butterfly stroke. Eur J Appl Physiol 2005; 93: $519-23$.
[3] Holmér I. Analysis of acceleration as a measure of swimming proficiency. In: Terauds J, Bedingfield E, Eds. Biomechanics and medicine in swimming III; 1978: Champain, Illianois:Human Kinetics Publishers 1979; pp. 119-25.

[4] Kolmogorov S, Duplischeva O. Active drag, useful mechanical power output and hydrodynamic force coefficient in different swimming strokes at maximal velocity. J Biomech 1992; 25: 311-18.

[5] Hermens H, Freriks B, Merletti R, et al., Ed. European recommendations for surface electromyography. Netherlands, Roessingh Research and Development 1999

[6] Carvalho J, Gonçalves P, Conceição F, Vilas-Boas JP. Eléctrodos Activos para EMG Diferencial de Superfície em Contexto Desportivo. Proceedings of the 1st Congresso Internacional de Ciências do Desporto; 1999: Porto: FADE-UP.

[7] Gonçalves P, Pereira S, Vilar S., et al. Underwater electromyograph system and his dialog with other instrumentation. Port J Sports Sci 2006; 6 (1): 21

[8] De Leva P. Adjustments to Zatsiorsky-Seluyanov's segment inertia parameters. J Biomech 1996; 29: 1223-30.

[9] Abdel-Aziz YI, Karara HM. Direct linear transformation from comparator coordinates into object space coordinates in close range photogrammetry. In: Proceedings of the American Society of Photogrammetry Symposium on Close Range Photogrammetry; Falls Church, American Society of Photogrammetry 1971; pp 1-18.

[10] Winter A. Biomechanics and motor control of human movement. 3rd ed. New York: Wiley 2005. 